\title{
The Orbitofrontal Cortex Gray Matter Is Associated With the Interaction Between Insomnia and Depression
}

\author{
Siyi Yu ${ }^{1 \dagger}$, Zhifu Shen ${ }^{1 \dagger}$, Rui Lai ${ }^{2}$, Fen Feng ${ }^{3}$, Baojun Guo ${ }^{1}$, Zhengyan Wang ${ }^{4}$, Jie Yang ${ }^{1}$, \\ Youping $\mathrm{Hu}^{1 *}$ and Liang Gong ${ }^{5,6 *}$ \\ 'Department of Acupuncture \& Tuina, Chengdu University of Traditional Chinese Medicine, Chengdu, China, ${ }^{2}$ Department of \\ Anesthesiology, People's Hospital of Deyang, Deyang, China, ${ }^{3}$ Affiliated Hospital of Chengdu University of Traditional Chinese \\ Medicine, Chengdu, China, ${ }^{4}$ Department of Pain Management, Sichuan Integrative Medicine Hospital, Chengdu, China, \\ ${ }^{5}$ Department of Neurology, Chengdu Second People's Hospital, Chengdu, China, ${ }^{6}$ Department of Neurology, Affiliated \\ ZhongDa Hospital, School of Medicine, Southeast University, Nanjing, China
}

Insomnia and depression are highly comorbid symptoms in both primary insomnia (PI) and major depressive disorder (MDD). In the current study, we aimed at exploring both the homogeneous and heterogeneous brain structure alteration in $\mathrm{PI}$ and MDD patients. Sixty-five MDD patients and 67 matched PI patients were recruited and underwent

OPEN ACCESS

Edited by:

Yu-Tao Xiang,

University of Macau, China

Reviewed by:

Yang-Kun Chen,

Dongguan People's Hospital, China

Huajun Liang,

University of Maryland, United States

*Correspondence:

Youping $\mathrm{Hu}$

hypcdutcm@yeah.net

Liang Gong

seugongliang@hotmail.com

†These authors have contributed equally to this work

Specialty section

This article was submitted to Mood and Anxiety Disorders,

a section of the journal

Frontiers in Psychiatry

Received: 02 June 2018 Accepted: 16 November 2018 Published: 04 December 2018

Citation:

Yu S, Shen Z, Lai R, Feng F, Guo B, Wang Z, Yang J, Hu Y and Gong L

(2018) The Orbitofrontal Cortex Gray Matter Is Associated With the

Interaction Between Insomnia and Depression. Front. Psychiatry 9:651. doi: 10.3389/fpsyt.2018.00651 a structural MRI scan. The subjects were sub-divided into four groups, namely MDD patients with higher or lower insomnia, and PI patients with higher or lower severe depression. A general linear model was employed to explore the changes in cortical thickness and volume as a result of depression or insomnia, and their interaction. In addition, partial correlation analysis was conducted to detect the clinical significance of the altered brain structural regions. A main effect of depression on cortical thickness was seen in the superior parietal lobe, middle cingulate cortex, and parahippocampal gyrus, while a main effect of insomnia on cortical thickness was found in the posterior cingulate cortex. Importantly, the interaction between depression and insomnia was associated with decreased gray matter volume in the right orbitofrontal cortex, i.e., patients with co-occurring depression and insomnia showed smaller brain volume in the right orbitofrontal cortex when compared to patients with lower insomnia/depression. These findings highlighted the role of the orbitofrontal cortex in the neuropathology of the comorbidity of insomnia and depression. Our findings provide new insights into the understanding of the brain mechanism underlying comorbidity of insomnia and depression.

Keywords: insomnia, depression, comorbidity, structural MRI, orbitofrontal cortex

\section{INTRODUCTION}

Insomnia represents a common symptom seen in the world population (about 30\%), with about $6-10 \%$ of the adult population reaching the diagnostic criteria for Primary insomnia (PI) (1). In contrast, major depressive disorder (MDD) is the second cause of disability worldwide, which is to become the world's most frequent and economically burdensome illness by $2030(2,3)$. Sleep complaints, especially the symptom of insomnia, are reported in up to $90 \%$ of MDD patients and can profoundly impact both the severity of depression and the course of the illness $(4,5)$. In addition, about $20 \%$ of patients with insomnia suffer from depression $(6,7)$. Furthermore, 
insomnia was found to be a predictor of depression given that non-depressed people with insomnia have a 2 -fold risk to develop depression according to a recent meta-analysis of longitudinal epidemiological studies (8). These findings suggest that the link between insomnia and depression is bidirectional. In addition, both antidepressants and hypnotic medication are commonly prescribed to patients with the combined condition of depression and insomnia $(9,10)$. Therefore, due to the heterogeneity and homogeneity between insomnia and depression, exploring the common and different brain mechanisms underlying such symptoms may help refine existing depression and insomnia treatments and develop personalized treatment for PI and MDD.

In the last decades, accumulating neuroimaging studies suggested PI and MDD to be associated with some functional and structural alterations in the brain of patients $(11,12)$. For example, Winkelman et al. found that patients with chronic PI reported an increased cortical volume in the rostral anterior cingulate cortex (rACC) when compared to normal sleepers, which was an indication of clinical severity (13). Other studies also reported volumetric differences in the frontal cortex, OFC, parietal cortex, precuneus and hippocampus $(14,15)$. In contrast, neuroimaging studies in patients with MDD described smaller volumes of the hippocampus, thalamus, insula, frontal lobe, orbitofrontal cortex and $\operatorname{rACC}(16,17)$. However, due to the heterogeneity between the two diseases, such results regarding the structural brain alteration were not always consistent $(11,18)$. More recently, an increasing number of researchers pay attention on both the common and different brain mechanisms underlying insomnia and depression. For example, Cheng et al. conducted a study on a bit sample of healthy individuals and found an increased functional connectivity in the lateral orbitofrontal cortex (OFC), dorsolateral prefrontal cortex, anterior and posterior cingulate cortex and insula, which was associated with both sleep and depressive scores (19). Furthermore, Liu et al. reported increased amplitude of low-frequency fluctuations (ALFF) during the resting state in the right inferior frontal gyrus and anterior insula in MDD patient with insomnia when compared to MDD patients without insomnia. In addition, they suggested that the abnormal ALFF was associated with sleep disturbance scores (20). Moreover, Li et al. found that patients with PI had reduced gray matter volume in the middle cingulate cortex, which was significantly associated with self-rating for depression score (21). Yang et al. suggested that decreased gray matter volume both in the left lingual gyrus and cerebellum predicts insomnia in female MDD patients (22). Considering the high comorbidity of insomnia and depression in the two neuropsychiatric disorders, only a few study have investigated their interaction effect on brain structure in both PI and MDD patients.

In the present study, we aimed at exploring the potential brain mechanism underlying the comorbidity of insomnia and depression using structural magnetic resonance imaging (MRI). First, we detected the main and the interaction effects of insomnia and depression on brain cortical thickness and volume in four heterogeneous subgroups of patients, i.e., MDD patients with higher or lower insomnia (MDD-HI or MDD-LI), and PI patients with higher or lower depression (PI-HD or PI-LD). Second, we explored the clinical association between the influenced brain regions in each group. Based on previous neuroimaging finding on PI and $\operatorname{MDD}(21,23,24)$, we hypothesized that the prefrontal cortex, especially the OFC and the anterior cingulate cortex, would be influenced by the interaction between insomnia and depression.

\section{METHODS AND MATERIALS}

\section{Participants}

The present study is a preliminary and retrospective research, the enrollment is separately for MDD and PI group. All participants were recruited from the outpatient of department of neurology and psychiatry of the Chengdu University of Traditional Chinese Medicine (CDUTCM). We selected sixty-five MDD patients and 67 age-, gender-, and education-matched PI patients (Table 1). This study was approved by the Research Ethics Committee of CDUTCM and all participants gave written informed consent. The following eligibility criteria were considered for MDD patients: (1) Met the diagnostic criteria for MDD according to the Diagnostic Statistical Manual of Mental Disorder, fourth Edition (DSM-IV); (2) the Hamilton Rating Scale for Depression-17 (HAMD) score was equal or above 17; (3) Naïve to antidepressant medications or a washout period of at least five half-lives of the previously prescribed medicine was undergone $(25,26) ;(4)$ Age between 18 and 55; and (5) Age at onset was $<50$ years. The following inclusion criteria were considered for PI patients: (1) Met the diagnostic criteria for PI according to the DSM-IV; (2) complaints of difficulty of falling asleep, maintaining sleep or early awakening for at least 3 months; (3) Age between 18 and 55; and (4) Age at onset was $<50$ years. The exclusion criteria for all patients included: (1) a history of other major psychiatric disorders or a neurological illness history, except for anxiety in the current state; (2) substance abuse, including caffeine, nicotine, and alcohol (27); (3) any brain lesions found by a T2 MRI scan.

\section{Behavior Assessment and Subgroup Division}

All participants underwent both a clinical and a behavioral assessment, while a neuropsychiatric examination was performed by two experienced neurologists (SY and ZS) who reached a consensus diagnosis. The HAMD for depression severity, the Hamilton Rating Scale for Anxiety (HAMA) for anxiety evaluation, and the HAMD sleep subscale (HAMD-S) for insomnia evaluation were used for assessing MDD patients. Following, according to the HAMD-S score, the MDD group was divided into the MDD subgroup with higher insomnia (MDDHI, HAMD-S score $>3$ ) and the MDD subgroup with lower insomnia (MDD-LD, HAMD-S score <3) $(20,28,29)$. Given that $5 \mathrm{MDD}$ patients reported a HAMD-S score equal to 3, they were excluded from the statistical analysis. In contrast, the Pittsburgh Sleep Quality Index (PSQI) for evaluating the insomnia severity (30), the self-rating depression scale (SDS) for depression severity, and the self-rating anxiety scale (SAS) for anxiety severity evaluation were used for assessing PI patients. Following, the PI group was also divided into two subgroups 
TABLE 1 | Demographic and clinical characteristics for all participants.

\begin{tabular}{|c|c|c|c|c|c|c|}
\hline Characteristic & PI-HD ( $n=29)$ & PI-LD $(n=28)$ & MDD-HI $(n=32)$ & MDD-LI $(n=28)$ & $F / T / \chi^{2}$ value & $p$-Value \\
\hline Age & $39.24 \pm 10.96$ & $38.42 \pm 11.86$ & $39.21 \pm 13.81$ & $35.67 \pm 10.37$ & 0.592 & 0.622 \\
\hline Gender (male/female) & $10 / 19$ & $10 / 18$ & $15 / 17$ & $10 / 18$ & 0.217 & $0.641^{\dagger}$ \\
\hline Education (years) & $12.96 \pm 3.98$ & $13.65 \pm 3.73$ & $12.09 \pm 3.42$ & $12.96 \pm 2.83$ & 1.93 & 0.129 \\
\hline eTIV (ml) & $1,484.48 \pm 126.93$ & $1,502.34 \pm 132.57$ & $1,480.17 \pm 146.82$ & $1,485.93 \pm 152.39$ & 0.173 & 0.915 \\
\hline Duration (months) & $82.17 \pm 82.85$ & $41.10 \pm 33.48$ & $78.10 \pm 89.12$ & $75.41 \pm 106.28$ & 2.78 & 0.007 \\
\hline PSQl & $14.58 \pm 2.13$ & $13.52 \pm 1.72$ & - & - & 2.25 & 0.028 \\
\hline HAMD-S & - & - & $4.91 \pm 0.82$ & $1.78 \pm 1.20$ & 11.91 & 0.000 \\
\hline SDS & $61.00 \pm 5.50$ & $46.32 \pm 3.67$ & - & - & 11.78 & 0.000 \\
\hline HAMD & - & - & $23.20 \pm 4.35$ & $16.36 \pm 5.72$ & 5.24 & 0.000 \\
\hline SAS & $54.62 \pm 6.09$ & $52.65 \pm 4.40$ & - & - & 1.53 & 0.131 \\
\hline HAMA & - & - & $17.40 \pm 5.95$ & $14.85 \pm 5.79$ & 1.67 & 0.101 \\
\hline
\end{tabular}

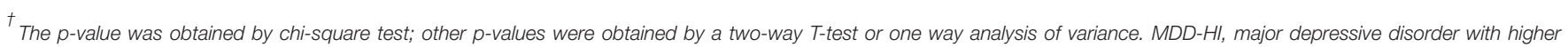

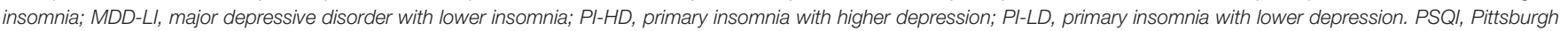

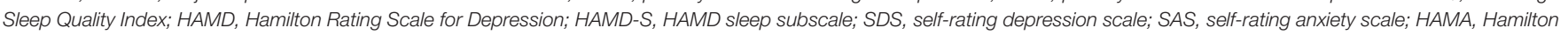
Rating Scale for Anxiety.

according to the SDS scores, namely the PI subgroup with higher depression (PI-HD, SDS score $>55$ ) and the PI subgroup with lower depression (PI-LD, SDS score $<50)$. Given that 10 patients reported to have a SDS score between 50 and 55, they were not included in the statistical analysis (31).

\section{Image Data Acquisition and Processing}

All participants underwent MRI scanning on the same 3.0-Tesla magnetic resonance scanner (Discovery MR750, General Electric, Milwaukee, WI, USA) equipped with a standard head coil. All participants were instructed not to consume caffeine, alcohol, or any other psychoactive substance in the $48 \mathrm{~h}$ prior to the scan. Tight however comfortable foam padding was used to minimize head motion, and earplugs were employed to reduce the scanner noise. Sagittal 3D T1-weighted images were acquired using a brain volume sequence with the following parameters: repetition time $(\mathrm{TR})=8.16 \mathrm{~ms}$, echo time $(\mathrm{TE})=3.18 \mathrm{~ms}$, flip angle $(\mathrm{FA})$ $=7^{\circ}$, field of view $(\mathrm{FOV})=256 \times 256 \mathrm{~mm}^{2}$, matrix $=256 \times 256$; slice thickness $=1 \mathrm{~mm}$, no gap; and 188 sagittal slices. During the MRI examination, all subjects were instructed to relax with their eyes closed without falling asleep or thinking about something. All participants were checked for their wakening status following the scan and they all claimed to be awake during the course of the study.

The images were processed using the standard surfacebased workflows in the FreeSurfer version 6.0 (http://surfer. nmr.mgh.harvard.edu/fswiki/recon-all/), including motion correction, non-parametric non-uniform intensity correction, intensity normalization, skull strip, automatic subcortical segmentation, white matter segmentation, tessellation, original surface smoothing, inflation, automatic topology fixer, surface registration, and cortical parcellation (32). The resulting surface reconstruction was visually inspected and manually edited in the following trouble shooting step (https://surfer.nmr.mgh.harvard. edu/fswiki/FsTutorial/TroubleshootingDataV6.0) by a single rater, blind to the diagnostic status. The troubleshooting included skull strip errors, segmentation errors, intensity normalization error, pial surface misplacement, and topological defect. The estimated Total Intracranial Volume (eTIV) was extracted as a covariate in a later analysis (33).

\section{Statistical Analysis Demographic and Behavioral Data}

One-way analysis of variance (ANOVA), independent samples $T$-tests and chi-square tests were conducted using the Statistical Package for the Social Sciences version 20.0 (SPSS, Inc., Chicago, IL, USA) to compare the demographic and behavioral data among groups. The Pearson correlation was employed to examine the relationships between HAMD/SDS scores and HAMD-S/PSQI scores. Considering the difference in both the disease duration between the two PI subgroups and in the HAMD score between the two MDD subgroups, the Pearson Correlation analysis was also conducted to investigate the relationships between both disease duration and SDS score in the PI groups and HAMD-S scores in the MDD groups. The significance was set at $p<0.05$.

\section{Structural Imaging}

A general linear model (GLM) analysis was employed to explore the effect of depression, insomnia, and their interaction on the reconstructed cortical surface image (mri_glmfit, FreeSurfer), regressed out the effect of gender, age, years of education, and the eTIV. The design matrices were created by a FreeSurfer Group Descriptor File (http://surfer.nmr. mgh.harvard.edu/fswiki/Fsgdf4G1V). For the main effect of depression, we set the MDD-HI, MDD-LI, and PI-HD as depressed patients, PI-LD as non-depressed patients; For the main effect of insomnia, we set the MDD-HI, PI-LD, PI-HD as insomnia patients, MDD-LI as non-insomnia patients; For the interaction of depression and insomnia effect, we set the MDD$\mathrm{HI}, \mathrm{PI}-\mathrm{HD}$ as the interaction of depression and insomnia. The the 
contrast matrix is set as the equation below:

$$
\begin{aligned}
Y_{i} & =\beta_{0}+\beta_{1} * \text { Gender }+\beta_{2} * \text { Age }+\beta_{3} * \text { Education }+\beta_{4} * e T I V \\
& +\beta_{5} * \text { Duration }+\beta_{6} * \text { Ins }+\beta_{7} * \text { Dep }+\beta_{8} *(\text { Ins } * \text { Dep })+\varepsilon
\end{aligned}
$$

where $\mathrm{Y}_{i}$ represents the thickness or volume of each vertex in cortex of the $i$ th participant; $\beta_{0}$ is the intercept of the straightline fitting in the model; $\beta_{1}, \beta_{2}, \beta_{3}, \beta_{4}$, and $\beta_{5}$ stand for the main effect of gender, age, education, eTIV volume, and disease duration, respectively, which were discarded as covariates of no interest in the GLM models. $\beta_{5}$ represents the main effect of depression, $\beta_{6}$ is the main effect of insomnia, $\beta_{7}$ describes the interaction effect of depression and insomnia. The error term $\varepsilon$ was assumed to have a Gaussian distribution so that no correlation across participants was shown.

After the GLM analyses, a cluster-wise correction for multiple comparison was processed using the Monte Carlo Simulation (mri_glmfit-sim, FreeSurfer, the vertex-wise threshold is $p<0.0001$, the cluster-wise $p<0.05$, iteration is 10,000 , adjust $p$-values for two hemispheres) (34).

\section{The Relationship Between Structural Imaging Features and Behavior}

To further detect whether depression and insomnia influenced the brain structural regions associated with clinical characteristics in both the MDD and PI groups, a Partial correlation analysis was conducted, after controlling for the effect of age, gender, years of education, disease duration and eTIV. The significance was set at $p<0.05$, and the false discovery rate (FDR) correction was used for multiple comparison correction.

\section{RESULTS}

\section{Demographic Information and Clinical Characteristics}

Significant differences in age, gender, years of education and eTIV were not observed between the four groups. As Table 1 illustrates, the disease duration in the PI-LD subgroup is shorter when compared to other groups. In addition, the PSQI score in the PILD subgroup is lower than the one in the PI-HD subgroup $(t=$ $2.25, p=0.02$ ), while the HAMD score in the MDD-HL subgroup is higher when compared to the one in the MDD-LI subgroup $(t=5.24, p<0.001)$. In contrast, a significant difference in the anxiety symptom was not found between the two PI and MDD groups. In the MDD group, the HAMD score was positively correlated with both the HAMA score $(r=0.46, p<0.001)$, and the HAMD-S score $(r=0.57, p<0.001)$. In concordance, the PSQI score was positively correlated with the SDS score in the PI group $(r=0.27, p=0.027)$. Significant relationships between disease duration, PSQI and SAS scores were not seen in PI groups. An absence of other significant relationships between the clinical characteristics was present.

\section{Main Effect of Depression on Brain Structure}

As illustrated in Figure 1 and Table 2, a main effect of depression on cortical thickness was seen in the bilateral superior parietal lobule (left SPL, $2.39 \pm 0.16$ vs. $2.45 \pm 0.10$; right SPL, $2.37 \pm$ 0.12 vs. $2.43 \pm 0.11$ ), the right middle cingulate cortex (MCC, $2.38 \pm 0.15$ vs. $2.46 \pm 0.12$ ), and the right parahippocampal gyrus $(2.42 \pm 0.18$ vs. $2.45 \pm 0.16)$, after adjusting for the effects of gender, age, and years of education and TIV. The cortical regions affected by depression were thinner in patients with depression when compared to patients with an absence of severe depression symptom. In contrast, a significant effect of depression on cortical volume was not found.

\section{Main Effect of Insomnia on Brain Structure}

After adjusting for the effects of gender, age, and years of education, a main effect of insomnia on cortical thickness was observed in the right PCC $(2.39 \pm 0.16$ vs. $2.43 \pm 0.14)$. As showed in Figure 2 and Table 2, the right PCC was thinner in patients with insomnia when compared to patients with an absence of severe insomnia symptom. In contrast, a significant effect of insomnia on cortical volume was not seen.

\section{Interaction Effect of Depression and Insomnia on Brain Structure}

A significant interaction effect of depression and insomnia on the right orbital frontal cortex (OFC) volume was detected, after both a flexible multiple comparison correction $(p<0.05)$ and controlling for the effects of gender, age, and years of education. As Figure 3 displays, patients with co-occurring depression and insomnia showed smaller brain volume in the right OFC when compared to patients without severe insomnia/depression, in both the MDD and the PI groups. A significant interaction effect of depression and insomnia on cortical thickness was not present after multiple comparison correction.

\section{Correlation Analysis}

An absence of significant FDR-adjusted correlations between influenced brain regions and relative clinical traits in the MDD and PI groups was reported.

\section{DISCUSSION}

To our knowledge, the present study was the first attempt to explore the interaction of insomnia and depression on brain structure in both PI and MDD patients. Three major findings were reported. First, insomnia and depression symptoms were closely associated in both the PI and MDD patients groups. Second, depression influences the brain structure in the SPL, MCC, and parahippocampal gyrus, while insomnia mainly influences the PCC thickness. Third, depression and insomnia interaction contributes to cortical volume loss in the right OFC. These findings indicated that the OFC might be a core region for the neuropathological alteration in comorbidity of insomnia and depression. Therefore, our findings provide new insights into the understanding of the brain mechanism underlying comorbidity of insomnia and depression.

The clinical features of the four subgroups verified the close association and the comorbidity between MDD and PI. In fact, patients with insomnia in the MDD group showed more severe depression, which is consistent with a previous 

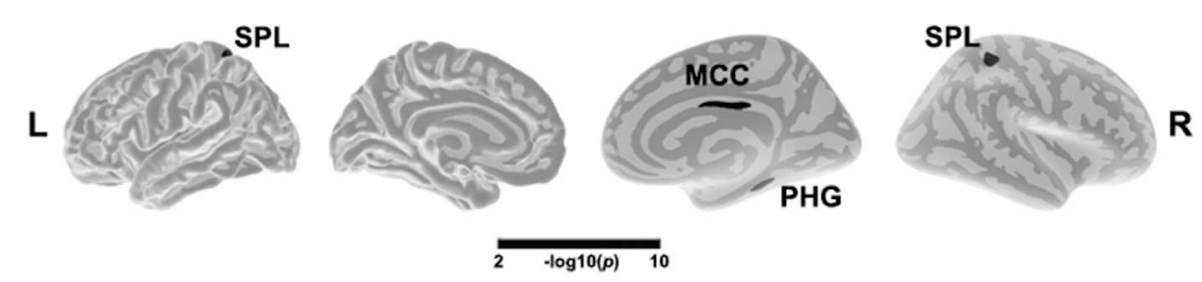

FIGURE 1 | Main effect of depression in cortical thickness across all patients. The color bar indicates the -log10(p) value after clusterwise correction for multiple comparisons using Monte Carlo simulations (vertex $p<0.0001$, cluster $p<0.05$ ). L, left; R, right; MCC, middle cingulate cortex; SPL, superior parietal lobule; PHG, parahippocampal gyrus.

TABLE 2 | The depression and insomnia and their interactive effects on cortical thickness and volume.

\begin{tabular}{|c|c|c|c|c|c|c|c|}
\hline Clusters & Max $T$ value & VtxMax & TalX & TalY & TalZ & CWP & NVtxs \\
\hline \multicolumn{8}{|c|}{ MAIN EFFECT OF DEPRESSION ON CORTICAL THICKNESS } \\
\hline 1. Left superior parietal lobe & 7.419 & 133,615 & -16.9 & -50.6 & 61.6 & 0.0058 & 292 \\
\hline 2. Right superior parietal lobe & 5.136 & 14,752 & 28.8 & -39.5 & 490.0 & 0.0006 & 563 \\
\hline 3. Right middle cingulate cortex & 5.948 & 35,246 & 3.3 & -7.3 & 29.5 & 0.0020 & 357 \\
\hline 4. Right parahippocampal gyrus & 5.825 & 113,996 & 35.8 & -35.0 & -13.8 & 0.0020 & 341 \\
\hline \multicolumn{8}{|c|}{ MAIN EFFECT OF INSOMNIA ON CORTICAL THICKNESS } \\
\hline 1. Right posterior cingulate cortex & 6.709 & 24,061 & 5.8 & -38.4 & 24.3 & 0.0026 & 346 \\
\hline \multicolumn{8}{|c|}{ INTERACTIVE EFFECT OF DEPRESSIVE AND INSOMNIA IN CORTICAL VOLUME* } \\
\hline 1. Right orbital frontal cortex & 3.615 & 139,780 & 6.9 & 0.0 & 64.8 & 0.0002 & 3,508 \\
\hline
\end{tabular}

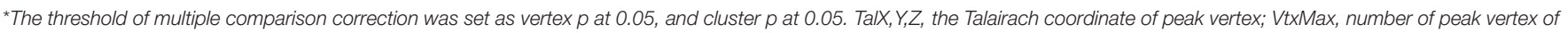
the significant cluster; CWP, cluster-wise probability and the nominal p-value; NVtxs, number of vertices in cluster.

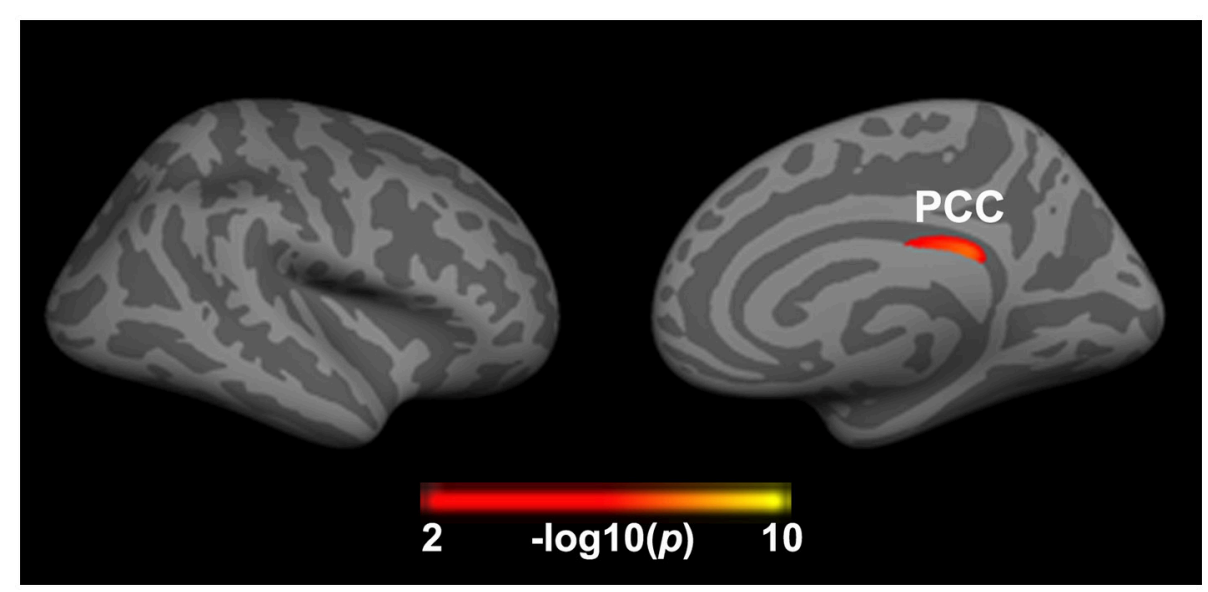

FIGURE 2 | Significant effect of insomnia on right cortical thickness across all patients. The color bar indicates the -log10(p) value after clusterwise correction for multiple comparisons using Monte Carlo simulations (vertex $p<0.0001$, cluster $p<0.05$ ). PCC, posterior cingulate cortex.

epidemiologic study (35). In concordance, PI patients with depression have a longer disease duration and more severe insomnia, which is positively associated with depression but not with the anxiety symptom. These results were proved also consistent with previous epidemiologic results on the long-term consequences of insomnia (1). The clinical results presented here support the notion that the relationship between insomnia and depression is bidirectional, and this relationship can change over time following disease progression or release (7).
In the present study, we investigated the insomnia and depression effects on brain structure in a pooled patient group. This approach enables, in fact, the detection of the insomnia/depression effect in two patient groups and might reveal the common neurobiology underlying such symptoms. Only cortical thickness was significantly influenced by insomnia and depression in the pooled population. Patients with depression showed thinning of the SPL, MCC, and parahippocampal gyrus. The SPL is involved in spatial cognition 

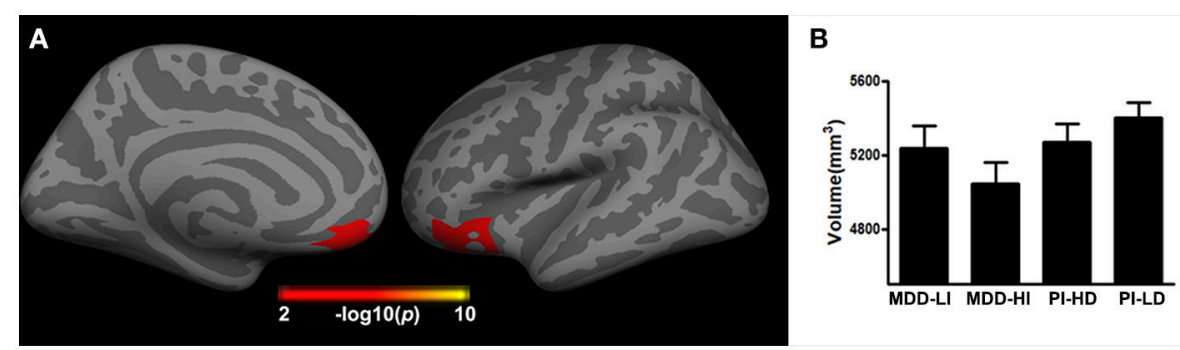

FIGURE 3 | The interactive effect of depression and insomnia on right cortical volume across all patients. (A) The interactive effect was found in right orbital frontal cortex; (B) The histogram indicated that the joint depression and insomnia showed smaller brain volume in the right orbital frontal cortex.

and sensorimotor integration $(36,37)$; previous studies also reported decreased gray matter volume and reduced functional connectivity in the SPL in MDD patients $(38,39)$. Although the alteration of the MCC is less commonly reported in depression studies, the posterior MCC is involved in attentional control during emotion regulation and showed a functional abnormality in MDD patients $(40,41)$. The present result provides a new insight into emotion regulation abnormality in patient with depression. Last, the parahippocampal gyrus is important for episodic memory and emotion cues processing (42); the thinning parahippocampal gyrus here found is consistent with previous structural imaging results in MDD patients (43), and indicated a common structural basis underlying both emotion and episodic memory processing in the patients with depression. Regarding patients with insomnia, the common structural alteration was seen in PCC, which is the core region of the default mode network and has been considered to be involved in emotion, memory and intrinsic control, and to be represent a neural substrate for human awareness (44, 45). The metabolism, functional connectivity abnormality and structural connectivity of the anterior default mode network was also reported in PI patients $(46,47)$. Generally, our findings on the main effect of insomnia and depression highlighted the morphological alteration in the thickness of the SPL, MCC, and parahippocampal gyrus in depression and of the PCC in insomnia.

Our most important finding was the localization of the insomnia/depression interaction effect on brain structure in the right OFC. The orbitofrontal area is involved in emotion, especially in reward processing, decision-making, and problemsolving abilities $(48,49)$. Previously, gray matter decrements in the OFC were reported in insomnia and depression patients, separately $(50,51)$. The decreased PFC volume may explain the insufficient decision-making and problem-solving abilities, and the attenuated recognition of environmental temperature in insomnia patients $(24,52)$. In addition, the reduced OFC volume may account for the distorted emotional stimuli evaluation, abnormal emotional and visceral regulation, anhedonia symptoms as well $(23,50)$. Interestingly, we found that co-occurrence of insomnia and depression is related to the worst volume decrease seen in the orbitofrontal cortex, especially, in MDD patients with insomnia. Baglioni and Riemann proposed a hypothesis stating that the link between persistent insomnia and depression is the alteration of the arousal system and its subsequent impact on affective and cognitive systems (53). Given that OFC receives widespread projections from arousal systems, including thalamus and amygdala (54), the decreased OFC volume may indicate an abnormal top-down control mechanism for arousal systems and dysfunctional emotional regulation and reward processing in patients with comorbidity of insomnia and depression. Overall, our results suggested that OFC is an essential area underlying the neuropathological mechanism of the comorbidity of insomnia and depression.

Two factors are likely to account for the absence in clinical significance of the alteration in cortical morphology of regions. First, considering that the behavioral assessments of depression and insomnia are not conformed between groups, we can only conduct the correlation analysis separately. Second, given that the clinical characteristics were robustly associated with demographic factors and relative stability of brain structure, some recent studies found that cortical structure feature is a relatively weak discriminant factor for current episode clinical status $(55,56)$. Further studies should include detailed and conformed neuropsychological tests, and a larger sample to verify this.

The limitations of the current study will now be highlighted. First, the present study is a preliminary and retrospective research, so the present study did not use the same estimating tool for depression and insomnia, we cannot explore the relationship between the influenced structural region and the clinical traits in a pooled group. In addition, the HAMD subscale of insomnia scores only has three items to estimate the insomnia, the HAMD total score is positively correlated with HAMD-S score, so it's difficult to completely remove the depression severity effect in the data analysis. These limitations regarding the clinical evaluation restricted the elaboration of our findings on brain structure. Second, not only insomnia, but also hypersomnia, is present in MDD patients (5); therefore, future studies should explore the brain mechanism underlying hypersomnia in MDD patients. Third, significant differences were found between the PI-HD and PI-LD groups in the PSQI total score, and between the MDD$\mathrm{HI}$ and MDD-LI groups in the HAMD total score, which might bias the findings to uncertain extent. Fourth, the present study strictly focused on the structural change of cortical regions in depression and insomnia, whereas the subcortical and cerebellar regions were unexplored. Future studies should explore these issues further. Fifth, the present study did not include a healthy 
control group, which again limits the discussion of our findings. Lastly, considering that our study had a cross-sectional design with relatively small sample sizes, future research using larger sample sizes, data pooling, and longitudinal designs is deemed necessary.

In conclusion, our present results indicated that the OFC may be a core region for the neuropathological mechanism in comorbidity of insomnia and depression. In addition, our findings provide new insights into the understanding of the brain mechanism underlying comorbidity of insomnia and depression and the development of rational treatment strategies for these patients.

\section{AUTHOR CONTRIBUTIONS}

SY, YH, and LG contributed to the conception and design of study. RL, BG, ZW, and FF contributed to the clinical

\section{REFERENCES}

1. Morin CM, Jarrin DC. Epidemiology of insomnia. prevalence, course, risk factors, and public health burden. Sleep Med Clin. (2013) 8:281-97. doi: 10.1016/j.jsmc.2013.05.002

2. Ferrari AJ, Charlson FJ, Norman RE, Patten SB, Freedman G, Murray CJ, et al. Burden of depressive disorders by country, sex, age, and year: findings from the global burden of disease study 2010. PLoS Med. (2013) 10:e1001547. doi: 10.1371/journal.pmed.1001547

3. Mathers CD, Loncar D. Projections of global mortality and burden of disease from 2002 to 2030. PLoS Med. (2006) 3:e442. doi: 10.1371/journal.pmed.0030442

4. Tsuno N, Besset A, Ritchie K. Sleep and depression. J Clin Psychiatry (2005) 66:1254-69. doi: 10.4088/JCP.v66n1008

5. Geoffroy PA, Hoertel N, Etain B, Bellivier F, Delorme R, Limosin $\mathrm{F}$, et al. Insomnia and hypersomnia in major depressive episode: prevalence, sociodemographic characteristics and psychiatric comorbidity in a population-based study. J Affect Disord. (2018) 226:132-41. doi: 10.1016/j.jad.2017.09.032

6. Soldatos CR. Insomnia in relation to depression and anxiety: epidemiologic considerations. J Psychosom Res. (1994) 38 (Suppl. 1):3-8.

7. Staner L. Comorbidity of insomnia and depression. Sleep Med Rev. (2010) 14:35-46. doi: 10.1016/j.smrv.2009.09.003

8. Baglioni C, Battagliese G, Feige B, Spiegelhalder K, Nissen C, Voderholzer $\mathrm{U}$, et al. Insomnia as a predictor of depression: a meta-analytic evaluation of longitudinal epidemiological studies. J Affect Disord. (2011) 135:10-9. doi: 10.1016/j.jad.2011.01.011

9. Jindal RD, Thase ME. Treatment of insomnia associated with clinical depression. Sleep Med Rev. (2004) 8:19-30. doi: 10.1016/S1087-0792(03)00025-X

10. Thase ME. Depression and sleep: pathophysiology and treatment. Dialogues Clin Neurosci. (2006) 8:217-26.

11. O'Byrne JN, Berman Rosa M, Gouin JP, Dang-Vu TT. Neuroimaging findings in primary insomnia. Pathol Biol. (Paris) (2014) 62:262-9. doi: 10.1016/j.patbio.2014.05.013

12. Jarnum H, Eskildsen SF, Steffensen EG, Lundbye-Christensen S, Simonsen CW, Thomsen IS, et al. Longitudinal MRI study of cortical thickness, perfusion, and metabolite levels in major depressive disorder. Acta Psychiatr Scand. (2011) 124:435-46. doi: 10.1111/j.1600-0447.2011.01766.x

13. Winkelman JW, Plante DT, Schoerning L, Benson K, Buxton OM, O’Connor $\mathrm{SP}$, et al. Increased rostral anterior cingulate cortex volume in chronic primary insomnia. Sleep (2013) 36:991-8. doi: 10.5665/sleep.2794

14. Winkelman JW, Benson KL, Buxton OM, Lyoo IK, Yoon S, O'Connor S, et al. Lack of hippocampal volume differences in primary insomnia and good estimate acquisition of imaging data. SY and LG performed imaging data analysis. SY and ZS wrote the manuscript. ZS and JY contributed revising the manuscript logically for important theoretical content. All authors contributed to the manuscript and have approved the final manuscript.

\section{FUNDING}

This work was supported by the programs of the National Natural Science Foundation of China (81303057, 81503670, and 81373560); The Science and Technology Department of Sichuan Province (2018JY0249).

\section{ACKNOWLEDGMENTS}

We thank all the participants involved in the study. We thank Jiaojian Wang for the help of statistical analysis of GLM.

sleeper controls: an MRI volumetric study at 3 Tesla. Sleep Med. (2010) 11:576-82. doi: 10.1016/j.sleep.2010.03.009

15. Joo EY, Noh HJ, Kim JS, Koo DL, Kim D, Hwang KJ, et al. Brain gray matter deficits in patients with chronic primary insomnia. Sleep (2013) 36:999-1007. doi: $10.5665 /$ sleep. 2796

16. Wagner G, Koch K, Schachtzabel C, Schultz CC, Sauer H, Schlosser RG. Structural brain alterations in patients with major depressive disorder and high risk for suicide: evidence for a distinct neurobiological entity? Neuroimage (2011) 54:1607-14. doi: 10.1016/j.neuroimage.2010.08.082

17. Kempton MJ, Salvador Z, Munafo MR, Geddes JR, Simmons A, Frangou S, et al. Structural neuroimaging studies in major depressive disorder. Metaanalysis and comparison with bipolar disorder. Arch Gen Psychiatry (2011) 68:675-90. doi: 10.1001/archgenpsychiatry.2011.60

18. Bora E, Fornito A, Pantelis C, Yucel M. Gray matter abnormalities in major depressive disorder: a meta-analysis of voxel based morphometry studies. J Affect Disord. (2012) 138:9-18. doi: 10.1016/j.jad.2011.03.049

19. Cheng W, Rolls ET, Ruan H, Feng J. Functional connectivities in the brain that mediate the association between depressive problems and sleep quality. JAMA Psychiatry (2018) 75:1052-61. doi: 10.1001/jamapsychiatry.2018.1941

20. Liu C-H, Guo J, Lu S-L, Tang L-R, Fan J, Wang C-Y, et al. Increased salience network activity in patients with insomnia complaints in major depressive disorder. Front Psychiatry (2018) 9:93. doi: 10.3389/fpsyt.2018.00093

21. Li M, Yan J, Li S, Wang T, Wen H, Yin Y, et al. Altered gray matter volume in primary insomnia patients: a DARTEL-VBM study. Brain Imaging Behav. (2018). doi: 10.1007/s11682-018-9844-x. [Epub ahead of print].

22. Yang X, Peng Z, Ma X, Meng Y, Li M, Zhang J, et al. Sex differences in the clinical characteristics and brain gray matter volume alterations in unmedicated patients with major depressive disorder. Sci Rep. (2017) 7:2515. doi: 10.1038/s41598-017-02828-4

23. Drevets WC. Orbitofrontal cortex function and structure in depression. Ann N Y Acad Sci. (2007) 1121:499-527. doi: 10.1196/annals.1401.029

24. Altena E, Vrenken H, Van Der Werf YD, van den Heuvel OA, Van Someren EJ. Reduced orbitofrontal and parietal gray matter in chronic insomnia: a voxel-based morphometric study. Biol Psychiatry (2010) 67:182-5. doi: 10.1016/j.biopsych.2009.08.003

25. Korgaonkar MS, Fornito A, Williams LM, Grieve SM. Abnormal structural networks characterize major depressive disorder: a connectome analysis. Biol Psychiatry (2014) 76:567-74. doi: 10.1016/j.biopsych.2014.02.018

26. Williams LM, Rush AJ, Koslow SH, Wisniewski SR, Cooper NJ, Nemeroff $\mathrm{CB}$, et al. International study to predict optimized treatment for depression (iSPOT-D), a randomized clinical trial: rationale and protocol. Trials (2011) 12:4. doi: $10.1186 / 1745-6215-12-4$

27. Martin LM, Triscari R, Boisvert R, Hipp K, Gersten J, West RC, et al. Development and evaluation of the lifestyle history questionnaire (LHQ) for 
people entering treatment for substance addictions. Am J Occup Ther. (2015) 69:6903250010p1-9. doi: 10.5014/ajot.2015.014050

28. Park S-C, Kim J-M, Jun T-Y, Lee M-S, Kim J-B, Jeong S-H, et al. Prevalence and clinical correlates of insomnia in depressive disorders: the CRESCEND study. Psychiatry Investig. (2013) 10:373-81. doi: 10.4306/pi.2013.10.4.373

29. Manber R, Blasey C, Arnow B, Markowitz JC, Thase ME, Rush AJ, et al. Assessing insomnia severity in depression: comparison of depression rating scales and sleep diaries. J Psychiatr Res. (2005) 39:481-8. doi: 10.1016/j.jpsychires.2004.12.003

30. Backhaus J, Junghanns $\mathrm{K}$, Broocks A, Riemann D, Hohagen F. Test-retest reliability and validity of the Pittsburgh sleep quality index in primary insomnia. J Psychosom Res. (2002) 53:737-40. doi: 10.1016/S0022-3999(02)00330-6

31. Su Q, Yu B, He H, Zhang Q, Meng G, Wu H, et al. Nut consumption is associated with depressive symptoms among chinese adults. Depress Anxiety (2016) 33:1065-72. doi: 10.1002/da.22516

32. Fischl B, Dale AM. Measuring the thickness of the human cerebral cortex from magnetic resonance images. Proc Natl Acad Sci USA. (2000) 97:11050-5. doi: $10.1073 /$ pnas. 200033797

33. Buckner RL, Head D, Parker J, Fotenos AF, Marcus D, Morris JC, et al. A unified approach for morphometric and functional data analysis in young, old, and demented adults using automated atlasbased head size normalization: reliability and validation against manual measurement of total intracranial volume. Neuroimage (2004) 23:724-38. doi: 10.1016/j.neuroimage.2004.06.018

34. Hagler DJ Jr., Saygin AP, Sereno MI. Smoothing and cluster thresholding for cortical surface-based group analysis of fMRI data. Neuroimage (2006) 33:1093-103. doi: 10.1016/j.neuroimage.2006.07.036

35. Soehner AM, Kaplan KA, Harvey AG. Prevalence and clinical correlates of co-occurring insomnia and hypersomnia symptoms in depression. J Affect Disord. (2014) 167:93-7. doi: 10.1016/j.jad.2014.05.060

36. Wolpert DM, Goodbody SJ, Husain M. Maintaining internal representations: the role of the human superior parietal lobe. Nat Neurosci. (1998) 1:529-33. doi: $10.1038 / 2245$

37. Koenigs M, Barbey AK, Postle BR, Grafman J. Superior parietal cortex is critical for the manipulation of information in working memory. J Neurosci. (2009) 29:14980-6. doi: 10.1523/JNEUROSCI.3706-09.2009

38. Zhao Y, Chen L, Zhang W, Xiao Y, Shah C, Zhu H, et al. Gray matter abnormalities in non-comorbid medication-naive patients with major depressive disorder or social anxiety disorder. EBio Med. (2017) 21:228-35. doi: 10.1016/j.ebiom.2017.06.013

39. Sacchet MD, Ho TC, Connolly CG, Tymofiyeva O, Lewinn KZ, Han LK, et al. Large-scale hypoconnectivity between resting-state functional networks in unmedicated adolescent major depressive disorder. Neuropsychopharmacology (2016) 41:2951-60. doi: 10.1038/npp.2016.76

40. Vogt BA. Midcingulate cortex: structure, connections, homologies, functions and diseases. J Chem Neuroanat. (2016) 74:28-46. doi: 10.1016/j.jchemneu.2016.01.010

41. Bertocci MA, Bebko GM, Mullin BC, Langenecker SA, Ladouceur $\mathrm{CD}$, Almeida JR, et al. Abnormal anterior cingulate cortical activity during emotional n-back task performance distinguishes bipolar from unipolar depressed females. Psychol Med. (2012) 42:1417-28. doi: 10.1017/S003329171100242X

42. Aminoff EM, Kveraga K, Bar M. The role of the parahippocampal cortex in cognition. Trends Cogn Sci. (2013) 17:379-90. doi: 10.1016/j.tics.2013. 06.009
43. Lener MS, Kundu P, Wong E, Dewilde KE, Tang CY, Balchandani P, et al. Cortical abnormalities and association with symptom dimensions across the depressive spectrum. J Affect Disord. (2016) 190:529-36. doi: 10.1016/j.jad.2015.10.027

44. Leech R, Sharp DJ. The role of the posterior cingulate cortex in cognition and disease. Brain (2014) 137:12-32. doi: 10.1093/brain/awt162

45. Vogt BA, Laureys S. Posterior cingulate, precuneal and retrosplenial cortices: cytology and components of the neural network correlates of consciousness. Prog Brain Res. (2005) 150:205-17. doi: 10.1016/S0079-6123(05)50015-3

46. Nie X, Shao Y, Liu SY, Li HJ, Wan AL, Nie S, et al. Functional connectivity of paired default mode network subregions in primary insomnia. Neuropsychiatr Dis Treat. (2015) 11:3085-93. doi: 10.2147/NDT.S95224

47. Kay DB, Karim HT, Soehner AM, Hasler BP, James JA, Germain A, et al. Subjective-objective sleep discrepancy is associated with alterations in regional glucose metabolism in patients with insomnia and good sleeper controls. Sleep (2017) 40:zsx155. doi: 10.1093/sleep/zsx155

48. Bechara A, Damasio H, Damasio AR. Emotion, decision making and the orbitofrontal cortex. Cereb Cortex (2000) 10:295-307. doi: 10.1093/cercor/10.3.295

49. Kringelbach ML. The human orbitofrontal cortex: linking reward to hedonic experience. Nat Rev Neurosci. (2005) 6:691-702. doi: 10.1038/nrn1747

50. Bremner JD, Vythilingam M, Vermetten E, Nazeer A, Adil J, Khan S, et al. Reduced volume of orbitofrontal cortex in major depression. Biol Psychiatry (2002) 51:273-9. doi: 10.1016/S0006-3223(01)01336-1

51. Koolschijn PC, van Haren NE, Lensvelt-Mulders GJ, Hulshoff Pol HE, Kahn RS. Brain volume abnormalities in major depressive disorder: a meta-analysis of magnetic resonance imaging studies. Hum Brain Mapp. (2009) 30:3719-35. doi: 10.1002/hbm.20801

52. Raymann RJ, Van Someren EJ. Diminished capability to recognize the optimal temperature for sleep initiation may contribute to poor sleep in elderly people. Sleep (2008) 31:1301-9.

53. Baglioni C, Riemann D. Is chronic insomnia a precursor to major depression? Epidemiological and biological findings. Curr Psychiatry Rep. (2012) 14:511-8. doi: 10.1007/s11920-012-0308-5

54. Rolls ET. The orbitofrontal cortex. Philos Trans R Soc Lond B Biol Sci. (1996) 351:1433-43. discussion: 43-4.

55. Perlman G, Bartlett E, DeLorenzo C, Weissman M, McGrath P, Ogden $\mathrm{T}$, et al. Cortical thickness is not associated with current depression in a clinical treatment study. Hum Brain Mapp. (2017) 38:4370-85. doi: 10.1002/hbm.23664

56. Yang XH, Wang Y, Huang J, Zhu CY, Liu XQ, Cheung EF, et al. Increased prefrontal and parietal cortical thickness does not correlate with anhedonia in patients with untreated first-episode major depressive disorders. Psychiatry Res. (2015) 234:144-51. doi: 10.1016/j.pscychresns.2015.09.014

Conflict of Interest Statement: The authors declare that the research was conducted in the absence of any commercial or financial relationships that could be construed as a potential conflict of interest.

Copyright (c) 2018 Yu, Shen, Lai, Feng, Guo, Wang, Yang, Hu and Gong. This is an open-access article distributed under the terms of the Creative Commons Attribution License (CC BY). The use, distribution or reproduction in other forums is permitted, provided the original author(s) and the copyright owner(s) are credited and that the original publication in this journal is cited, in accordance with accepted academic practice. No use, distribution or reproduction is permitted which does not comply with these terms. 